\title{
Effect of Liver Resection-induced Increases in Hepatic Venous Pressure Gradient on Development of Postoperative Acute Kidney Injury
}

Christian Reiterer ( $\square$ christian.reiterer@meduniwien.ac.at )

Department of Anaesthesia, Intensive Care Medicine and Pain Medicine, Medical University of Vienna Alexander Taschner

Department of Anaesthesia, Intensive Care Medicine and Pain Medicine, Medical University of Vienna Florian Luf

Department of Anaesthesia and Intensive Care, Hanusch Krankenhaus, Vienna

\section{Manfred Hecking}

Division of Nephrology and Dialysis, Dept. of Internal Medicine III, Medical University of Vienna

\section{Dietmar Tamandl}

Department of Biomedical Imaging and Image-guided Therapy, Medical University of Vienna

\section{Oliver Zotti}

Department of Anaesthesia, Intensive Care Medicine and Pain Medicine, Medical University of Vienna

\section{Thomas Reiberger}

Division of Gastroenterology and Hepatology, Dept. of Internal Medicine III, Medical University of Vienna

\section{Patrick Starlinger}

Department of Surgery, Medical University of Vienna

\section{Mattias Mandorfer}

Christian Doppler Laboratory for Portal Hypertension and Liver Fibrosis, Medical University of Vienna

\section{Edith Fleischmann}

Department of Anaesthesia, Intensive Care Medicine and Pain Medicine, Medical University of Vienna

\section{Research Article}

Keywords: acute kidney injury, liver resection, hepatic venous pressure gradient, renin-angiotensinaldosterone system

Posted Date: June 25th, 2021

DOl: https://doi.org/10.21203/rs.3.rs-622535/v1

License: (a) (i) This work is licensed under a Creative Commons Attribution 4.0 International License. Read Full License 
Version of Record: A version of this preprint was published at BMC Nephrology on January 8th, 2022. See the published version at https://doi.org/10.1186/s12882-021-02658-7. 


\section{Abstract}

Background: The impact of changes in portal pressure before and after liver resection (defined as $\triangle \mathrm{HVPG}$ ) on postoperative renal function remains unknown. Therefore, we investigated the effect of $\triangle H V P G$ on (i) the incidence of postoperative AKI and (ii) the renin-angiotensin system (RAAS) and sympathetic nervous system (SNS) activity.

Methods: We included 30 patients undergoing partial liver resection. Our primary outcome was postoperative AKI according to KDIGO criteria. For our secondary outcome we assessed the plasma renin, aldosterone, noradrenaline, adrenaline, dopamine and vasopressin concentrations prior and 2 hours after induction of anaesthesia, on the first and fifth postoperative day. HVPG was measured prior and immediately after liver resection.

Results: $\triangle \mathrm{HVPG}$ could be measured in 21 patients with 12 patients HVPG showing increases in HVPG $(\triangle H V P G \geq 1 \mathrm{mmHg})$ while 9 patients remained stable. AKI developed in $7 / 12$ of patients with increasing HVPG, but only in $2 / 9$ of patients with stable DHVPG $(p=0.302)$. Noradrenalin levels were significantly higher in patients with increasing DHVPG than in patients with stable DHVPG. $(p=0.009)$. Biomarkers reflecting RAAS and SNS activity remained similar in patients with increasing vs. stable DHVPG.

Conclusions: Patients with increasing HVPG were more likely to develop postoperative AKI as compared those with stable HVPG values.

\section{Introduction}

Acute kidney injury (AKI) occurs in about $6 \%$ of patients undergoing noncardiac surgery [1]. Moreover, after liver surgery the incidence reaches up to $15 \%$ [2]. The underlying pathophysiology of the high incidence of $\mathrm{AKI}$ in patients undergoing liver-resection is still not fully understood. A possible explanation might be the effect of liver resection on the intrahepatic vascular resistance (IVR). A postoperative increase of IVR might lead to an "acute hepatorenal like syndrome" affecting renal function due to the increased release of endogenous vasopressors resulting in renal arterial vasoconstriction [3-5].

Increased portal vein pressure caused by liver resection $[6,7]$ leads to splanchnic vasodilation and further reduce systemic vascular resistance [8]. This results in an activation of the sympathetic nervous and the renin-angiotensin system, in order to maintain an adequate organ perfusion pressure [9]. Furthermore, the activation of the neurohumoral system results in an excessive release of noradrenaline and angiotensin II [9]. These pathophysiologic mechanisms impair renal perfusion and reduce renal function [9].

In the non-operative setting, measurement of the hepatic venous pressure gradient (HVPG) is an established method to stratify liver pathophysiology in patients with cirrhosis [10]. However, there is scarce evidence regarding the impact HVPG changes in the perioperative period, specifically in patients without underlying cirrhosis undergoing liver resection. The difference between preoperative measured HVPG and postoperative measured HVPG during liver resection; which we defined as $\triangle H V P G$. 
In order to explain the higher incidence of AKI after liver resection, we tested the effect of $\triangle \mathrm{HVPG}$ on the incidence AKI as our primary outcome. We further evaluated the effect of $\triangle \mathrm{HVPG}$ on the activity of sympathetic nervous system and renin-angiotensin system as our secondary outcome.

\section{Methods}

This study was approved by the Institutional Review Board of the Medical University of Vienna (EK 424/2010) and registered at ClinicalTrials.gov (NCT01700231). We obtained written informed consent from all patients prior to enrollment. The study was conducted according to the "Declaration of Helsinki" and followed the ICH GCP Guidelines at the Medical University of Vienna. We included patients between 18 and 85 years with neoplastic liver tumors undergoing elective hepatic resection. Patients with a preoperative $H V P G>10 \mathrm{mmHg}$ and renal failure were excluded.

\section{Patient characteristics and recorded parameters}

Demographic data including age, sex, BMI, American Society of Anesthesiologists (ASA) physical status, comorbidities, type of surgery and preoperative laboratory values were recorded. We recorded routine intraoperative variables including time of anesthesia and surgery, and fluid and anesthesia management. We recorded esophageal doppler derived hemodynamic data including stroke volume and cardiac output. Intraoperative core temperature was measured and the distal esophagus.

Laboratory liver and renal function tests included: serum-creatinine, serum-albumin, prothrombin index and bilirubin. These were assessed before surgery and on the postoperative days 1 and 5 . Postoperative maximum concentration was defined as the maximum value measured within 2 hours after surgery, on the first and fifth postoperative day.

Acute kidney injury was diagnosed according to the Kidney Disease: Improving Global Outcome (KDIGO) definitions [11], considering the maximum increase during the first 5 postoperative days (PODs): $\Delta \mathrm{Cr}=$ Maximum ( $\left.\mathrm{Cr}_{\mathrm{POD} 1}, \mathrm{Cr}_{\mathrm{POD} 2}, \ldots, \mathrm{Cr}_{\mathrm{POD} 5}\right) / \mathrm{Cr}_{\text {Preop }}$

\section{Anaesthesia protocol}

For induction of anaesthesia 3-4 mg/ $\mathrm{kg}$ propofol, $2-3 \mu \mathrm{g} / \mathrm{kg}$ fentanyl and $0.6 \mathrm{mg} / \mathrm{kg}$ rocuronium was given. Anaesthesia was maintained with sevoflurane in $30 \%$ oxygen carrier. We administered additional boli of fentanyl or rocuronium according to the patient's requirements. Ventilatory rate was adjusted to maintain an end-tidal $\mathrm{P}_{\mathrm{CO} 2}$ of 35-40 mmHg. Normothermia was maintained with forced air warming. Standard monitoring included electrocardiography (ECG), invasive blood pressure and pulse oximetry. A central venous catheter was inserted after induction of anesthesia. Mean arterial pressure was maintained above $65 \mathrm{mmHg}$. We used an esophageal doppler (Cardiac Q, Deltex Medical Group PLC, Chichester, UK) for goal-directed fluid therapy according to a previously published protocol [12]. All patients were given $5-7 \mathrm{~mL} \mathrm{~kg}^{-1}$ of lactated Ringer solution during induction of anaesthesia and 
followed by $3-5 \mathrm{~mL} \mathrm{~kg}^{-1}$ per hour normalized to ideal body weight for maintenance throughout surgery. Red cells were transfused as necessary to maintain a hematocrit level above $26 \%$.

\section{Liver resection surgery}

Surgery was performed according to standardized operational protocol. We used a cavitron ultrasound surgical aspiration (Integra CUSA Excel) for liver parenchyma dissection. To coagulate the small vessels we used bipolar coagulation, while major vascular structures were ligated or sewed. The weight of the resected liver tissue was recorded.

\section{Invasive hemodynamic measurements}

Portal pressure is clinically evaluated by measuring the hepatic venous pressure gradient (HVPG), i.e. the difference between the wedged hepatic venous pressure and the free hepatic venous pressure, as previously described [10]. Briefly, a catheter introducer sheet is placed in the jugular vein using the Seldinger technique, and a main hepatic vein was cannulated under fluoroscopic guidance using a $7 \mathrm{~F}$ balloon-tipped catheter (Straight Occlusion Catheter, Medi-Tech; Boston Scientific Cork Ltd., Cork, Ireland). After fluoroscopic control of a sufficient wedge position in the hepatic vein, we measured free and wedged hepatic venous pressures in triplicates to calculate the HVPG. The inferior vena cava pressure was also recorded to rule out an increased post-hepatic pressure. $\triangle$ HVPG was defined as difference between the pre- and the postoperative HVPG. Patients were stratified according to the $\triangle H V P G$ into two groups as (i) $\triangle H V P G \geq 1 \mathrm{mmHg}$, defined as postoperative HVPG higher than preoperative HVPG and (ii) $\triangle H V P G \leq 0 \mathrm{mmHg}$ defined as postoperative HVPG was similar or lower than preoperative HVPG.

\section{Statistics}

Statistical analysis was performed with IBM SPSS Statistics (Version 25). Patient characteristics, demographic data, preoperative laboratory values, renal and liver specific data were compared for balance. Normal distribution of data was tested using a Kolmogorov-Smirnov test. Normally distributed data were presented as mean \pm standard deviation, not normally distributed data were given as median and percentile. Chi-square test was used to comparing categorical variables

We stratified patients into two groups: patients with $\Delta \mathrm{HVPG} \geq 1 \mathrm{mmHg}$ and patients with stable $\Delta \mathrm{HVPG}$. We compared the incidence of AKI between both groups using a Fisher exact test. Fluid management and hemodynamics were compared between both groups using a Student's test or a Mann-Whitney-U test as appropriate.

To determine the effect of $\triangle \mathrm{HVPG}$ on the postoperative sympathetic nervous system and reninangiotensin system activity we measured renin, aldosterone, noradrenalin, adrenalin, dopamine and vasopressin concentrations before, 2 hours after induction of anaesthesia, and on the first and fifth postoperative day. Postoperative maximum concentrations of renin, angiotensin, dopamine, noradrenalin and adrenaline were compared between the two groups using Mann-Whitney-U test. 
Postoperative maximum concentrations of hepatic and renal function tests including creatinine, albumin, prothrombin index and bilirubin were compared between both groups using a Mann-Whitney-U test. Variables were summarized as median [25th, 75th percentiles] or mean (SD). Categorical variables were summarized as frequency (percent). We used SPSS for statistical analysis. (Version 25, IBM SPSS Statistic, Armonk, NY, USA)

\section{Results}

We included 30 patients undergoing elective liver resection from October 2010 to July 2013 at the Medical University of Vienna. We were unable to measure the postoperative hepatic venous pressure in 9 patients. Therefore, these patients were excluded from the final analysis. Baseline characteristics and laboratory measurements were shown in the online supplements.

Baseline characteristics, demographics, comorbidities, type of surgery, and laboratory values were similar between both groups. (Table 1)

In 12 patients $\triangle H V P G$ was $\geq 1 \mathrm{mmHg}$ and in 9 patients $\Delta H V P G$ remained unchanged $(\Delta H V P G \leq 0)$. There was no difference in the incidence of AKI according to the KDIGO criteria between patients with $\Delta \mathrm{HVPG} \geq 1 \mathrm{mmHg}$ as compared to patients with unchanged $\Delta \mathrm{HVPG} \leq 0 \mathrm{mmHg}(p=0.302)$. (Table 2$)$ Postoperative maximum creatinine was significantly higher in patients with $\Delta \mathrm{HVPG} \geq 1 \mathrm{mmHg}$ as compared to those with $\Delta \mathrm{HVPG} \leq 0 \mathrm{mmHg} .(1.4 \mathrm{mg} / \mathrm{dL}[1.2 ; 1.7]$ and $1.1 \mathrm{mg} / \mathrm{dL}[1.2 ; 1.6] ; p=0.039)$ (Table 2)

Time of surgery and anesthesia, anaesthetic medication, intraoperative total fluid volume, urine output and blood loss did not differ between both groups. (Table 3) Intraoperative hemodynamic data were similar between both groups.

Differences in liver specific laboratory parameters are shown in table 4.

\section{Liver volumetric}

Preoperative liver volume did not differ between the stratified groups. Resected liver volume as well as tumor volume was significantly bigger in patients with $\Delta \mathrm{HVPG}$ was $\geq 1 \mathrm{mmHg}$ as compared to patients with $\triangle H V P G \leq 0 \mathrm{mmHg}$. The remaining postoperative liver volume was significantly smaller in patients with $\triangle H V P G$ was $\geq 1 \mathrm{mmHg}$. (Table 2)

\section{Catecholamines}

Maximum postoperative noradrenalin concentration was significantly higher in patients with $\triangle \mathrm{HVPG}$ was $\geq 1 \mathrm{mmHg}$ as compared to patients with $\Delta \mathrm{HVPG} \leq 0 \mathrm{mmHg}(3534$ [2379; 19679] vs 1376 [803; 2953], $\mathrm{p}<$ 0.009). Postoperative maximum adrenalin concentration (258 [66; 443] vs 129 [85; 443], $p=0.568)$ and postoperative maximum dopamine concentration $(54[43 ; 134]$ vs $62[50 ; 69], p=0.653)$ remained similar between both groups. (Table 3) (Fig. 1a - c) 


\section{Renin-aldosterone-angiotensin-system}

There were no significant differences in RAAS specific biomarkers, such as renin, aldosterone and vasopressin between both study groups. (Table 3). (Fig. 2a - c).

\section{Discussion}

Our study strongly suggests that the incidence of postoperative AKI after liver resection is higher in patients with increasing $\triangle \mathrm{HVPG}$ (at least $\geq 1 \mathrm{mmHg}$ ) as compared to patients with unchanged $\Delta \mathrm{HVPG}$.

$58 \%$ of patients with $\triangle \mathrm{HVPG} \geq 1 \mathrm{mmHg}$ developed AKI in contrast to only $22 \%$ of patients with stable $\Delta \mathrm{HVPG} \leq 0 \mathrm{mmHg}$. The reported incidence of $\mathrm{AKI}$ in an unselected patient population undergoing noncardiac general surgery is approximately $10 \%$ and thus, considerably lower than in our study population [1]. Although the incidence of AKI did not differ significantly between both study groups, serum creatinine was significantly higher in patients with a $\triangle H V P G \geq 1 \mathrm{mmHg}$ after surgery and their postoperative creatinine values remained significantly higher.

A retrospective analysis in over 38,000 patients undergoing noncardiac surgery showed that even minor increases in serum creatinine values are associated with a two-fold increased risk of death [1]. Our data highlight that patients undergoing liver resection are more likely to develop postoperative complications due to impaired renal function. Therefore, postoperative renal function should be closely monitored in these patient population.

Furthermore, optimal intraoperative fluid management is important to maintain perioperative renal function. Restrictive fluid therapy during abdominal surgery was associated with an increased risk of acute kidney injury compared to a liberal fluid regimen [13]. In our trial we performed esophagus doppler guided goal-directed fluid management to optimize intraoperative volume status and to minimize the risk of hypovolemia. All intraoperative hemodynamic parameters including MAP, stroke volume and cardiac output were similar between patients with increasing $\Delta \mathrm{HVPG} \geq 1 \mathrm{mmHg}$ and patients with stable $\Delta \mathrm{HVPG}$ $\leq 0 \mathrm{mmHg}$. A potential bias related to differences in intraoperative fluid management on the incidence of postoperative AKI can therefore be excluded.

Interestingly, a retrospective analysis of 167 patients undergoing living liver donor hepatectomy showed no effect of surgery on postoperative serum creatinine and BUN concentrations within the first three postoperative days [14]. This could be explained by apparent differences between both study populations, since we included patients scheduled for liver resection due to malignant liver disease who often suffered from preexisting comorbidities, while the aforementioned retrospective study only enrolled healthy middle-aged patients scheduled for liver donation. The abnormal liver parenchyma in patients with liver disease-associated liver cancer likely further enhance (postoperative) intrahepatic vascular resistance. The acute reduction of hepatic vascular bed due to liver resection results in increased hepatic vascular resistance and lead simultaneously to a reduction of hepatic vascular compliance [15]. The diseased liver parenchyma combined with acute perturbation of hepatoportal hemodynamics caused by 
surgery might be main trigger factors for an increase in postoperative HVPG. In patients with liver metastasis or cirrhosis, arterial hepatic blood flow was significantly higher as compared to healthy volunteers [16]. This might be a sign of decreased hepatic vascular compliance in malignant liver diseases. Therefore, it seems likely that reduced vascular compliance in these patients undergoing liver resection results in an increased postoperative HVPG which ultimately affects renal function.

A portal venous pressure exceeding $5 \mathrm{mmHg}$ defines portal hypertension [17]. Although, the median increase in patients with increasing $\triangle \mathrm{HVPG}$ was only $2 \mathrm{mmHg}$, the incidence of postoperative AKI was up to $58 \%$. Patients undergoing liver resection due to malignant liver disease might, however, be more vulnerable to even small increases in HVPG, specifically when HVPG rises abruptly.

Increased portal venous pressure activate the hepatorenal reflex resulting in an impairment of renal physiology [18]. The activity of the SNS plays an important role in early and late hepatorenal disorders [19]. In patients with cirrhosis, increased plasma noradrenalin concentrations are common, which is explained by the enhanced SNS activity [20]. Interestingly, in our study only noradrenalin concentrations were significantly higher in patients with $\triangle H V P G \geq 1 \mathrm{mmHg}$ as compared in patients with $\triangle \mathrm{HVPG}$ $\leq 0 \mathrm{mmHg}$. Adrenaline and dopamine were not affected by postoperative $\triangle H V P G$. While several trials registered higher RAAS activity in patients with liver cirrhosis [21], we did not observe difference in RAAS activity between patients with increasing vs. stable postoperative HVPG. An potential explanation might be that the RAAS is more active in patients with long-standing cirrhosis with pronounced portal hypertension [22]. Consequently, RAAS specific biomarkers might be unable to assess the hepatorenal reflex in the immediate postoperative period. Noradrenalin on the other hand is released immediately from sympathetic nerve endings during stressful events and might therefore be more appropriate to assess the hepatorenal reflex and might further reflect systemic stress.

Preoperative estimation of functional liver reserve is important to predict postoperative liver failure in patients undergoing liver resection surgery $[23,24]$. Low Platelet count, decreased cholinesterase and increased bilirubin are strong predictors for impaired liver recovery after surgery [23, 24]. Estimated liver function in patients with increasing $\triangle H V P G \geq 1 \mathrm{mmHg}$ was significantly impaired and thus, these patients might be not able to compensate liver function after resection and counteract with an enhanced release of catecholamines, specifically noradrenalin. Noradrenaline is a strong alpha-receptor vasoconstrictor affecting renal blood flow [25]. Therefore, this could be another explanation for the higher incidence of $A K I$ in patients with $\triangle H V P G \geq 1 \mathrm{mmHg}$.

A limitation of this trial is the small number of patients included and thus, the interpretation of our results has to be done with some caution. Although noradrenaline was significantly higher in patients with $\triangle \mathrm{HVPG} \geq 1 \mathrm{mmHg}$, catecholamines have a short half-life of only a few minutes. Elevated concentrations in our study were measured during surgery and within 2 hours after surgery, therefor we cannot fully exclude the fact that this might be the stress response caused by surgery itself. In patients with $\Delta H V P G$ $\geq 1 \mathrm{mmHg}$ the resected liver volume was significantly larger than in the other group, and we unfortunately do not have information of long-term changes in $\triangle \mathrm{HVPG}$. 
In conclusion, our data demonstrate that patients undergoing liver resection who show an immediate postoperative increase in HVPG are at considerable risk to develop postoperative AKI and therefore renal function should be monitored very closely. Due to our small sample size, our investigation should be seen as a hypothesis generating study. This emphasizes that further research is needed to clarify the effect of postoperative increases in HVPG on renal function.

\section{Declarations}

1. Funding: No funds, grants, or other support was received.

2. Competing interests: The authors have no financial or non-financial competing interests.

3. Ethics approval: This study was performed in line with the principles of the Declaration of Helsinki. Approval was granted by the Institutional Review Board of the Medical University of Vienna (EK 424/2010) and registered at ClinicalTrials.gov (NCT01700231).

4. Availability of data and material: The datasets generated and analyzed during the current study are available from the corresponding author on reasonable request.

\section{References}

1. Kork, F. et al. Minor postoperative increases of creatinine are associated with higher mortality and longer hospital length of stay in surgical patients. Anesthesiology 123, 1301-1311 (2015). https://doi.org/10.1097/ALN.0000000000000891

2. Tomozawa, A., Ishikawa, S., Shiota, N., Cholvisudhi, P. \& Makita, K. Perioperative risk factors for acute kidney injury after liver resection surgery: an historical cohort study. Can. J. Anesth. 62, 753-761 (2015). https://doi.org/ï» ¿10.1007/s12630-015-0397-9

3. Schroeder, E., Shear, L., Sancetta, S. \& Gabuzda, G. Renal failure in patients with cirrhosis of the liver. Am. J. Med. 43, 887-896 (1967). https://doi.org/10.1016/0002-9343(67)90247-1

4. Lautt, W. W. Regulatory processes interacting to maintain hepatic blood flow constancy: vascular compliance, hepatic arterial buffer response, hepatorenal reflex, liver regeneration, escape from vasoconstriction. Hepatol. Res. 37, 891-903 (2007). https://doi.org/ï»i10.1111/j.1872034X.2007.00148.x

5. Moreau, R. \& Lebrec, D. Acute kidney injury: new concepts - hepatorenal syndrome: the role of vasopressors. Nephron - Physiol. 109, 73-79 (2008). https://doi.org/ï» ¿10.1159/000142939

6. Morsiani, E., Aleotti, A. \& Ricci, D. Haemodynamic and ultrastructural observations on the rat liver after two-thirds partial hepatectomy. J. Anat. 192, 507-515 (1998). https://doi.org/10.1046/j.14697580.1998.19240507.x

7. Doignon, I. et al. Immediate neuroendocrine signaling after partial hepatectomy through acute portal hyperpressure and cholestasis. J. Hepatol. 54, 481-488 (2011).

https://doi.org/10.1016/j.jhep.2010.07.012 
8. Martell, M., Coll, M., Ezkurdia, N., Raurell, I. \& Genescà, J. Physiopathology of splanchnic vasodilation in portal hypertension. World J. Hepatol. 2, 208-220 (2010). https://doi.org/10.4254/wjh.v2.i6.208

9. Stadlbauer, V. P. et al. Relationship between activation of the sympathetic nervous system and renal blood flow autoregulation in cirrhosis. Gastroenterology 134, 111-119 (2008). https://doi.org/10.1053/j.gastro.2007.10.055

10. Reiberger, T., Schwabl, P., Trauner, M., Peck-Radosavljevic, M. \& Mandorfer, M. Measurement of the hepatic venous pressure gradient and transjugular liver biopsy. J. Vis. Exp. 2020, 1-16 (2020). https://doi.org/10.3791/58819

11. Kellum, J. A. et al. Kidney disease: Improving global outcomes (KDIGO) acute kidney injury work group. KDIGO clinical practice guideline for acute kidney injury. Kidney Int. Suppl. 2, 1-138 (2012). https://doi.org/10.1038/kisup.2012.1

12. Gan, T. J. et al. Goal-directed intraoperative fluid administration reduces length of hospital stay after major surgery. Anesthesiology 97, 820-826 (2002). https://doi.org/10.1097/00000542-20021000000012

13. Myles, P. S. et al. Restrictive versus liberal fluid therapy for major abdominal surgery. N. Engl. J. Med. 378, 2263-2274 (2018). https://doi.org/10.1056/NEJMoa1801601

14. Wang, C. H. et al. Effect and outcome of intraoperative fluid restriction in living liver donor hepatectomy. Ann. Transplant. 22, 671-676 (2017). https://doi.org/10.12659/AOT.905612

15. Golriz, M. et al. Hepatic hemodynamic changes following stepwise liver resection. J. Gastrointest. Surg. 20, 587-594 (2016). https://doi.org/10.1007/s11605-015-3021-y

16. Leen, E. et al. Hepatic perfusion changes in patients with liver metastases: comparison with those patients with cirrhosis. Gut 34, 554-557 (1993). https://doi.org/10.1136/gut.34.4.554

17. Reiberger, T. et al. Austrian consensus guidelines on the management and treatment of portal hypertension (Billroth III). Wien. Klin. Wochenschr. 129, 135-158 (2017). https://doi.org/10.1007/s00508-017-1262-3

18. Jalan, R., Forrest, E. H., Redhead, D. N., Dillon, J. F. \& Hayes, P. C. Reduction in renal blood flow following acute increase in the portal pressure: evidence for the existence of a hepatorenal reflex in man? Gut 40, 664-670 (1997). https://doi.org/10.1136/gut.40.5.664

19. Henriksen, J. H. \& Ring-Larsen, H. Hepatorenal disorders: role of the sympathetic nervous system. Semin. Liver Dis. 14, 35-43 (1994). https://doi.org/10.1055/s-2007-1007296

20. Henriksen, J. H., Ring-Larsen, H., Kanstrup, I. L. \& Christensen, N. J. Splanchnic and renal elimination and release of catecholamines in cirrhosis. evidence of enhanced sympathetic nervous activity in patients with decompensated cirrhosis. Gut 25, 1034-1043 (1984). https://doi.org/10.1136/gut.25.10.1034

21. Paternostro, R. et al. Plasma renin concentration represents an independent risk factor for mortality and is associated with liver dysfunction in patients with cirrhosis. J. Gastroenterol. Hepatol. 32, 184190 (2017). https://doi.org/10.1111/jgh.13439 
22. Casey, S. et al. Activation of the alternate renin-angiotensin system correlates with the clinical status in human cirrhosis and corrects post Liver transplantation. J. Clin. Med. 8, 1-13 (2019). https://doi.org/10.3390/jcm8040419

23. Torzilli, G. et al. Hepatectomy for stage $B$ and stage $C$ hepatocellular carcinoma in the barcelona clinic liver cancer classification: results of a prospective analysis. Arch. Surg. 143, 1082-1090 (2008). https://doi.org/10.1001/archsurg.143.11.1082

24. Donadon, M. et al. Potential role of cholinesterases to predict short-term outcome after hepatic resection for hepatocellular carcinoma. Updates Surg. 65, 11-18 (2013). https://doi.org/10.1007/s13304-012-0174-z

25. Hoogenberg, K., Smit, A. J. \& Girbes, A. R. J. Effects of low-dose dopamine on renal and systemic hemodynamics during incremental norepinephrine infusion in healthy volunteers. Crit. Care Med. 26, 260-265 (1998). https://doi.org/10.1097/00003246-199802000-00022

\section{Tables}


$\Delta \mathrm{HVPG} \geq 1 \mathrm{mmHg} \quad \Delta \mathrm{HVPG} £ 0 \mathrm{mmHg} \quad p$-Value $(\mathrm{n}=12) \quad(\mathrm{n}=9)$

\begin{tabular}{llllll} 
Age, $y r s$ & 73 & {$[62,79]$} & 70 & {$[52,73]$} & 0.027 \\
\hline Height, $\mathrm{cm}$ & 172 & $(7)$ & 171 & $(11)$ & 0.841 \\
\hline Weight, kg & 74 & $(13)$ & 79 & $(18)$ & 0.458 \\
Sex, no. (\%) & & & & & \\
\hline Men & 2 & $(17)$ & 6 & $(67)$ & 0.375 \\
\hline Woman & 10 & $(83)$ & 3 & $(33)$ & \\
\hline
\end{tabular}

Comorbidities, no. (\%)

\begin{tabular}{llllll}
\hline Arterial Hypertension & 5 & $(42)$ & 2 & $(29)$ & 0.373 \\
\hline NIDDM & 1 & $(0)$ & 1 & $(14)$ & 0.667 \\
\hline Pulmonary & 0 & $(0)$ & 2 & $(29)$ & 0.076 \\
\hline NASH & 4 & $(33)$ & 2 & $(29)$ & 0.488 \\
\hline Type of Surgery, no. $(\%)$ & & & & & 0.005 \\
\hline Heminepatectomy & 10 & $(83)$ & 2 & $(22)$ & \\
\hline Partial Liver Resection & 2 & $(17)$ & 7 & $(78)$ & \\
\hline Laboratory Parameters & & & & & \\
\hline Bilirubin, $m g / d L$ & 1 & {$[0.7 ; 1.4]$} & 0.5 & {$[0.4 ; 0.7]$} & 0.002 \\
\hline ASAT, $U / L$ & 49 & {$[23 ; 82]$} & 44 & {$[24 ; 28]$} & 0.336 \\
\hline ALAT, $U / L$ & 35 & {$[21 ; 61]$} & 27 & {$[14 ; 45]$} & 0.546 \\
\hline Creatinine, $m g / d L$ & 0.8 & $(0.2)$ & 0.9 & $(0.1)$ & 0.819 \\
\hline CRP, $m g / d L$ & 0.2 & {$[0.1 ; 0.3]$} & 0.7 & {$[0.1 ; 0.8]$} & 0.722 \\
\hline Albumin, $g / L$ & 34.0 & $(3)$ & 36.0 & $(4)$ & 0.728 \\
\hline Prothrombin Time, \% & 73 & $(11)$ & 87 & $(16)$ & 0.259 \\
\hline Cholinesterase, $k U / L$ & 4.9 & $(1.0)$ & 6.8 & $(1.5)$ & 0.108 \\
\hline Platelets, G/dL & 137 & $(37)$ & 190 & $(46)$ & 0.018 \\
\hline vWF, \% & 176 & {$[114 ; 200]$} & 186 & {$[159 ; 285]$} & 1.000 \\
\hline RiCo, \% & 154 & $(49)$ & 153 & $(61)$ & 0.606 \\
\hline
\end{tabular}


$\begin{array}{llllll}\text { Fibrinogen, } m g / d L & 300 & 348 & (68) & 348\end{array}$

Summary statistics of patient characteristics are presented as counts, percentages of patients, means $( \pm S D)$, and median $\left[25^{\text {th }}\right.$ percentile, $75^{\text {th }}$ percentile]. All $P$-values are for unpaired Student's- $t$ tests, MannWhitney-U test or chi-square tests as appropriate. NIDDM, non-insulin dependent diabetes mellitus; NASH; non-alcoholic steatohepatitis; ASAT, aspartate aminotransaminase; ALAT, alanine aminotransaminase; CRP, C-reactive protein; vWF, von Willebrand factor; RiCo, Ristocetin cofactor 
Table 2: Outcome parameters

\begin{tabular}{|c|c|}
\hline$\Delta \mathrm{HVPG} \geq 1 \mathrm{mmHg}$ & $\Delta \mathrm{HVPG} £ 0 \mathrm{mmHg}$ \\
\hline$(n=12)$ & $(n=9)$ \\
\hline
\end{tabular}

Hepatic Venous Pressure Gradient

\begin{tabular}{|c|c|c|c|c|c|}
\hline Preoperative, $\mathrm{mmHg}$ & 3.5 & {$[2.0 ; 6.8]$} & 4.0 & $\begin{array}{l}{[2.0 ;} \\
7.0]\end{array}$ & 1.000 \\
\hline $\begin{array}{l}\text { Postoperative, } \\
\mathrm{mmHg}\end{array}$ & 6.5 & {$[4.0 ; 9.8]$} & 4.0 & $\begin{array}{l}{[2.0 ;} \\
6.5]\end{array}$ & 0.041 \\
\hline$\Delta \mathrm{HVPG}, \mathrm{mmHg}$ & 2.0 & {$[1.0 ; 3.8]$} & 0.0 & $\begin{array}{l}{[-1.0 ;} \\
0.0]\end{array}$ & $<.001$ \\
\hline
\end{tabular}

\section{Primary Outcome}

KDIGO, no. (\%)

0.302

None

5

(42)

7

(78)

I

4

2

II

2

(17)

0

III

1

(8)

0

$\Delta$ Creatinine, $m g / d L$

$1.4 \quad[1.2 ; 1.7]$

1.1

(0)

\section{Secondary Outcomes}

Renin-angiotensin-aldosteronesystem

\begin{tabular}{lllllll} 
Renin, $\mu / E / m L$ & Baseline & 7.8 & {$[2.2 ; 62.0]$} & 51.3 & {$[0.6 ; 63]$} & 1.000 \\
\hline Aldosterone, $p g / m L$ & Max & 29.5 & {$[8.6 ; 155.4]$} & 85.6 & {$[2.1 ; 296.6 \mid$} & 0.833 \\
& Baseline & 25 & {$[15 ; 170]$} & 53 & {$[35 ; 103]$} & 0463 \\
\hline Vasopressin, $p g / d L$ & Max & 169 & {$[28 ; 273]$} & 130 & {$[8 ; 253]$} & 0.970 \\
& Maxeline & 1.2 & {$[<1 ; 11.2]$} & $<1$ & {$[<1 ;<1]$} & 0.021 \\
\hline
\end{tabular}

Sympathetic Nervous System

$\begin{array}{lllllll}\text { Noradrenalin, } n g / d L & \text { Baseline } & 276 & {[213 ; 2431]} & 218 & {[113 ; 354]} & 0.200 \\ & \text { Max } & 3534 & {[2379 ;} & 1376 & {[803 ; 2953]} & 0.009 \\ & & & 19679] & & & \end{array}$

Adrenalin, $n g / d L$

Baseline $13 \quad[5 ; 62]$

$17 \quad[5 ; 46]$

0.595 


\begin{tabular}{lllllll}
\hline & Max & 258 & {$[66 ; 443]$} & 129 & {$[85 ; 443]$} & 0.568 \\
\hline Dopamine, $n g / d L$ & Baseline & 0 & {$[0 ; 13]$} & 5 & {$[0 ; 23]$} & 0.653 \\
\hline & Max & 54 & {$[43 ; 134]$} & 62 & {$[50 ; 69]$} & 0.653
\end{tabular}

Differences in the incidence of AKI stratified according to the KDIGO criteria are presented as counts and percentages of patients. Summary catecholamines and RAAS specific biomarkers are presented as medians [ $25^{\text {th }}$ percentile, $75^{\text {th }}$ percentile]. $p$-values are for chi-square or Mann-Whitney-U test as appropriate. KDIGO, kidney disease improving global outcomes; 
Table 3: Intraoperative characteristics

$$
\begin{array}{ll}
\Delta \mathrm{HVPG} \geq 1 \mathrm{mmHg} & \Delta \mathrm{HVPG} £ 0 \mathrm{mmHg} \quad p \text {-Value } \\
(\mathrm{n}=12) & (\mathrm{n}=9)
\end{array}
$$

Duration

$\begin{array}{llllll}\text { Anaesthesia, } \min & 300 & {[275 ; 360]} & 210 & {[181 ; 263]} & 0.046 \\ \text { Surgery, } \min & 240 & {[213 ; 264]} & 142 & {[125 ; 241]} & 0.019\end{array}$

Medication

Fentanyl, $\mu g$

Propofol, $m g$

Metronidazol, $m g$

Cefuroxim, $m g$

Fluid Management

Total Fluid, $m L$

Urine Output, $m L$

Blood Loss, $m L$

Hemodynamic

\begin{tabular}{l} 
MAP, $m m H g$ \\
SV, $m L$ \\
\hline
\end{tabular}

$\mathrm{CO}, \mathrm{mL} / \mathrm{min}$

4.3

CVP, $m m H g$

10

$0.04 \quad[0.03 ; 0.11]$

0

[0.0; 0.2]

(15)

$(1.0)$

(3)

5.4

10

2756

669

50

74

(7)

0.471

Noradrenalin, $m g$

Phenylephrine, mg

CT Liver Volumetrics

\begin{tabular}{llllll} 
Pre-OP Liver Volume, $m L$ & 1640 & {$[1385 ; 3119]$} & 1564 & {$[1270 ; 1793]$} & 0.374 \\
\hline Resected Liver Volume, $m L$ & 574 & {$[195 ; 787]$} & 84 & {$[64 ; 179]$} & 0.026 \\
\hline Post-OP Liver Volume, $m L$ & 897 & {$[706 ; 1255]$} & 1361 & {$[1137 ; 1735]$} & 0.003 \\
\hline Tumor Volume, $m L$ & 177 & {$[24 ; 1313]$} & 12 & {$[5 ; 62]$} & 0.033
\end{tabular}

Summary characteristics of intraoperative measurements presented as means (SD) or medians $\left[25^{\text {th }}\right.$ percentile, $75^{\text {th }}$ percentile]. All $P$-values are for unpaired Student's- $t$ tests or Mann-Whitney-U tests as 
appropriate. MAP, mean arterial pressure; SV, stroke volume; CO, cardiac output; CVP, central venous pressure; CT, computer tomography;

Table 4: Postoperative maximum liver specific parameters

\begin{tabular}{llllll}
\hline & \multicolumn{2}{l}{$\begin{array}{l}\Delta \text { HVPG } \geq 1 \mathrm{mmHg} \\
(\mathrm{n}=12)\end{array}$} & \multicolumn{2}{l}{$\begin{array}{l}\Delta \text { HVPG } £ 0 \mathrm{mmHg} \\
(\mathrm{n}=9)\end{array}$} & $p$-Value \\
\hline Bilirubin, $m g / d L$ & 2.4 & {$[1.4 ; 2.8]$} & 1.2 & {$[0.8 ; 1.2]$} & 0.030 \\
\hline ASAT, $U / L$ & 836 & {$[276 ; 1466]$} & 389 & {$[188 ; 352]$} & 0.188 \\
\hline ALAT, $U / L$ & 671 & {$[214 ; 1065]$} & 322 & {$[168 ; 440]$} & 0.256 \\
\hline Albumin, $g / L$ & 32 & $(4)$ & 33 & $(6)$ & 0.423 \\
\hline Prothrombin Time, \% & 72 & $(12)$ & 88 & $(13)$ & 0.007 \\
\hline Cholinesterase, $k U / L$ & 4.4 & $(0.8)$ & 6.1 & $(1.5)$ & 0.002 \\
\hline Platelets. $G / d L$ & 169 & $(48)$ & 196 & $(33)$ & 0.170 \\
\hline vWF, \% & 360 & {$[325 ; 412]$} & 325 & {$[290 ; 333]$} & 0.165 \\
\hline RiCo, \% & 433 & $(128)$ & 317 & $(85)$ & 0.030 \\
\hline Fibrinogen, $m g / d L$ & 370 & $(94)$ & 538 & $(125)$ & 0.002 \\
\hline
\end{tabular}

Summary characteristics of postoperative maximum liver specific laboratory concentration during the first five days after surgery. Measurements are presented medians $\left[25^{\text {th }}\right.$ percentile, $75^{\text {th }}$ percentile]. All $P$ values are for Mann-Whitney-U tests. ASAT, aspartate aminotransaminase; ALAT, alanine aminotransaminase; $v W F$, von Willebrand factor, RiCo, Ristocetin cofactor

\section{Figures}


a)

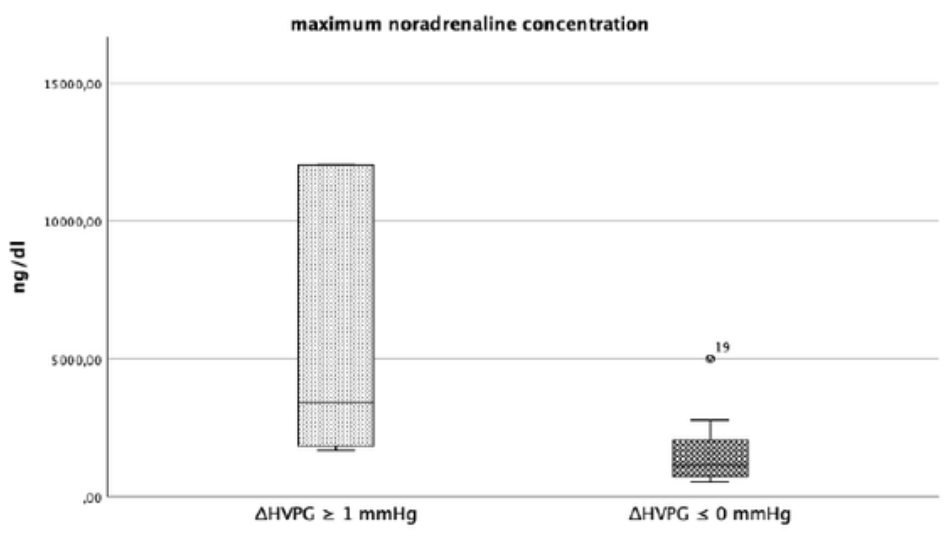

b)

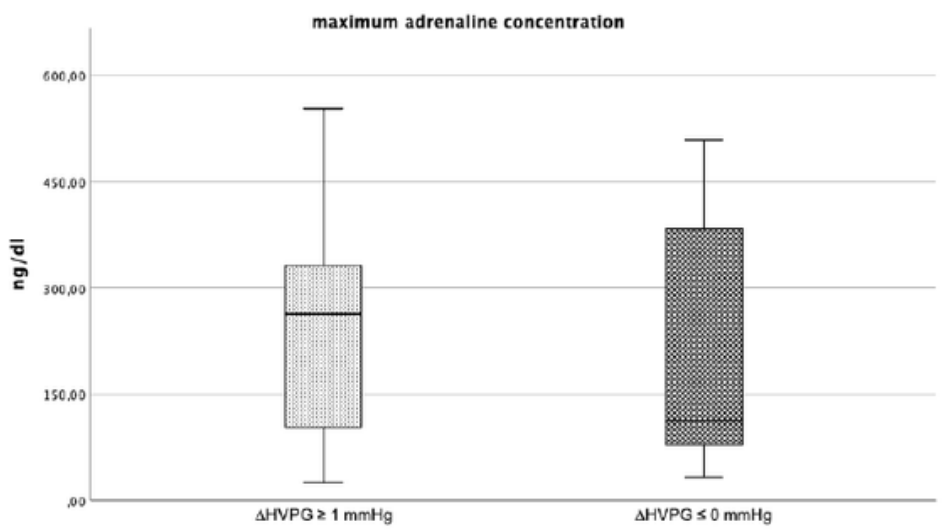

c)

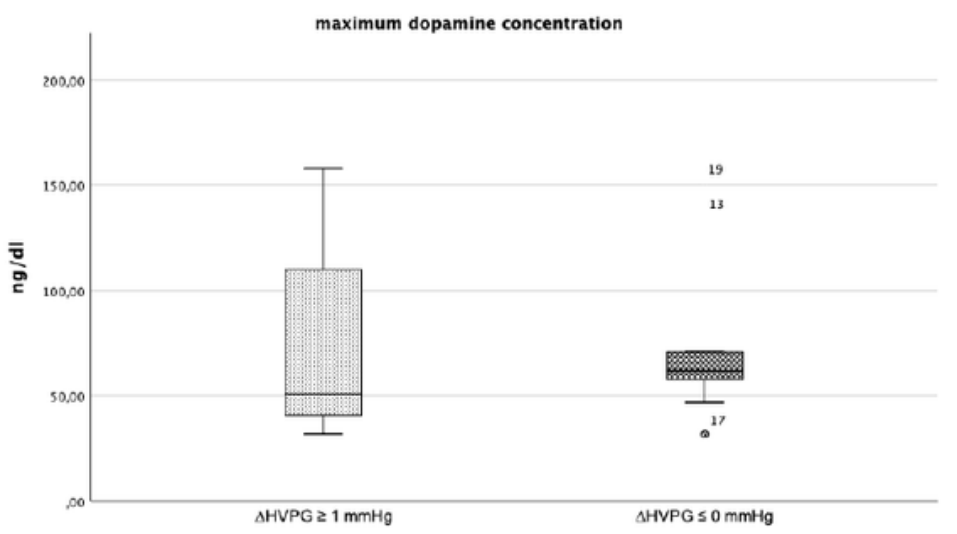

Figure 1

a-c: The following plots show boxplot of the maximum a) noradrenaline b) adrenaline and c) dopamine concentration between patients with $\Delta \mathrm{HVPG} \geq 1 \mathrm{mmHg}$ as compared to patients with $\triangle \mathrm{HVPG} \otimes 0 \mathrm{mmHg}$. 


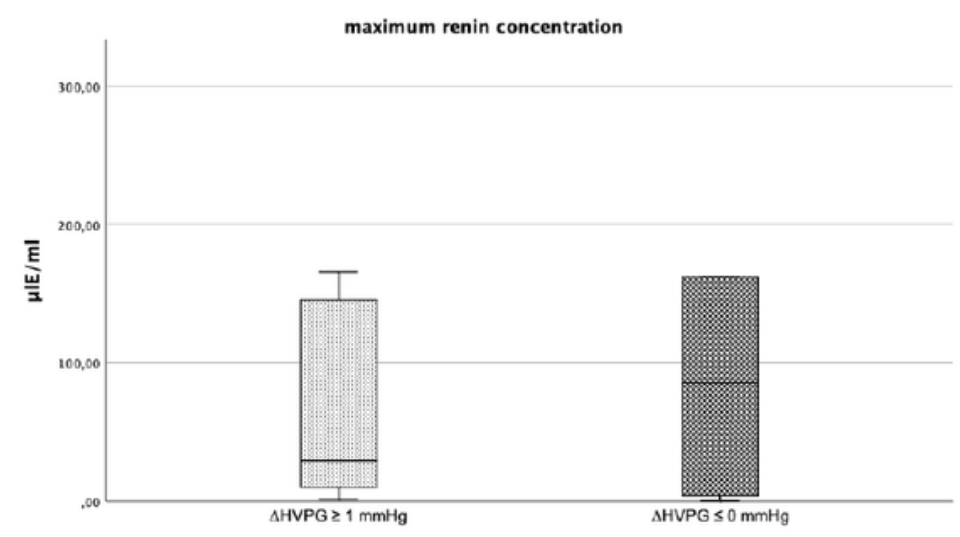

b)

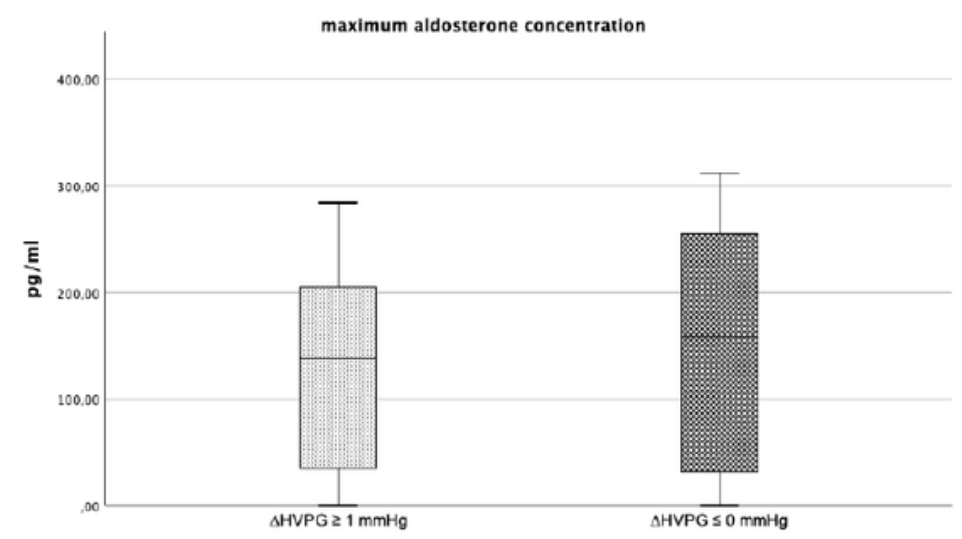

c)

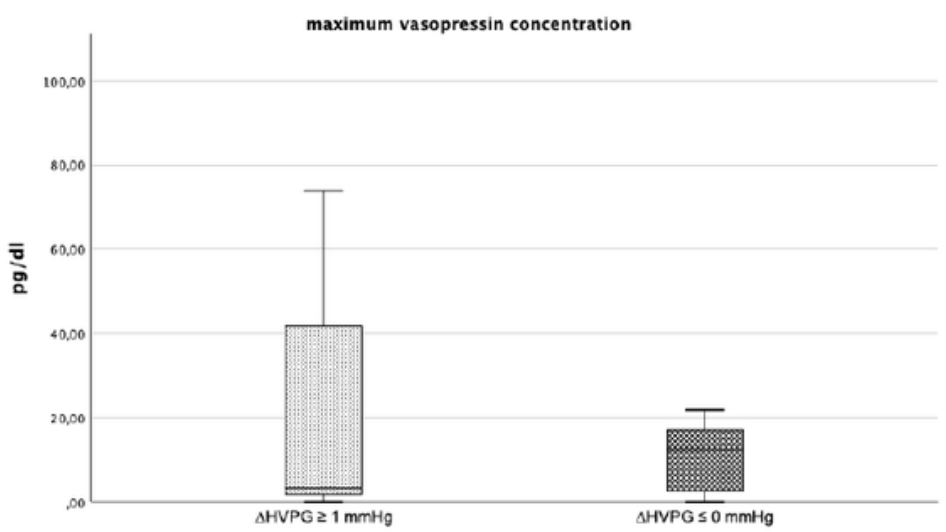

\section{Figure 2}

a-c: The following plots show boxplot of the maximum a) renin b) aldosterone and c) vasopressin concentration between patients with $\triangle \mathrm{HVPG} \geq 1 \mathrm{mmHg}$ as compared to patients with $\triangle \mathrm{HVPG} \otimes 0 \mathrm{mmHg}$. 